been collected so far. A full analysis of all the results will be presented.

Conclusion. The introduction of working time regulations such as the European Working Time Directive (2003) as well as local service reconfigurations leading to nurse-led liaison services and home treatment teams, have reduced the opportunity for trainees to undertake emergency assessments. Across the Severn Deanery, there is a discrepancy in the opportunity core trainees' have to undertake emergency assessments - depending on their rota, stage of training, and services available. This difference in trainee experience, depending on locality, has been further impacted by COVID-19 and the introduction of cohorted wards.

Trainees in Bath and Gloucester are predominantly covering the wards during on-calls and, therefore, we set out to ensure that they are regularly rostered to obtain emergency experience, helping them meet their core training competences. Initial results from two cycles of an emergency / out-of-hours experience rota suggest increased experience and confidence in first-line emergency assessments, enabling them to work towards meeting their core training requirements.

\section{West Midlands region less than full time training survey}

\section{Eleanor Parkinson* and Fiona Hynes}

Birmingham and Solihull Mental Health Foundation Trust ${ }^{*}$ Corresponding author.

doi: 10.1192/bjo.2021.423

Aims. To more fully understand the training experience of less than full time (LTFT) trainees working in psychiatry in the West Midlands Region with the aim of identifying areas that would improve the training experience.

Background. LTFT training has grown in popularity since its formal introduction in 2007. The greater participation of women in medicine and generational changes in lifestyle expectations are some of the factors behind this trend. Approximately 13\% of psychiatry trainees in the UK are training LTFT, bringing the benefit of allowing trainees to balance caring responsibilities or health conditions with continuing their postgraduate training. However it is not without its challenges for trainees which we aimed to explore in this survey.

Method. An electronic survey was sent out to all trainees via email, LTFT trainees of all training grades were invited to respond. Trainees were contacted in the five mental health trusts making up the region. The survey contained 32 questions that covered a range of topics including educational opportunities, perceived attitudes to LTFT trainees and training experience. Data were collected over a six month period in 2019. There were 22 responses to the survey region-wide.

Result. $86 \%$ of respondents were working reduced sessions in full-time posts with implications for their clinical workload and $14 \%$ responded that their clinical contact time was not adjusted to reflect their working hours. $36 \%$ of respondents experienced difficulties attending their formal teaching programme while $82 \%$ had attended educational commitments on non-working days. $14 \%$ of respondents felt training LTFT did not allow them to meet training requirements while $23 \%$ would not recommend LTFT training in the West Midlands to others. Trainees cited difficulties managing a full time workload and not having support from supervisors as reasons for these views. $40 \%$ of respondents reported experiencing negative attitudes from seniors and 50\% felt isolated from other trainees due to LTFT training status.
Conclusion. The survey has developed our understanding of the challenges faced by LTFT trainees and it has been communicated regionally and to employing trusts to promote action. For example, at a trust level, the use of personalised work schedules can address some common difficulties. More effectively communicating sources of support to trainees, sharing best practice and providing networking opportunities are suggested as next steps regionally. New administrative processes to maintain an accurate list of LTFT trainees is vital in implementing this. Improving the information given to trainers is another development area.

\section{Survey of junior doctors' perspective of serious incident reviews}

Olusegun Popoola ${ }^{1 \star}$, Kuben Naidoo ${ }^{1}$ and Amrith Shetty ${ }^{2}$

${ }^{1}$ Mersey Care NHS Foundation Trust and ${ }^{2}$ Cheshire and Wirral Partnership NHS Foundation Trust

${ }^{\star}$ Corresponding author.

doi: 10.1192/bjo.2021.424

Aims. Serious incidents according to NHS England (2015) are incidents where the consequences to patients, families and carers, staff or organisations are so significant or potential for learning are so great that a heightened response is justified. There is anectoctal evidence that this process is potentially difficult for junior doctors and the primary purpose of learning may be lost due to the stress involved.

Our aim was to evaluate junior doctors perspective of serious incident reviews. A secondary aim was to organise local and regional workshops based on the outcome of our findings to address misconceptions around serious incident investigations.

Method. A survey was developed using survey monkey and distributed to all trainees across the Mersey region through the Medical Education teams.

The junior doctors range from core trainees to higher trainees. The survey encouraged the use of free texting if necessary.

Results from the survey were then analysed

Result. 18 junior doctors across the 3 mental health Trusts in the Mersey region responded.

12 respondents have been involved in a serious incident investigation in the past and 9 of the respondents stated that they did not recieve any support during the process. Out of the 3 that were supported, one rated the support as poor and frightening.

$55.56 \%$ af all respondents found the process of serious incident reviews hard to understand.

$66 \%$ of all respondents admitted that they are aware that the purpose of the review is for learning purposes.

$100 \%$ of respondents agreed that a workshop to discuss the purpose and process of serious incidents investigation to aid their understanding would be useful.

Conclusion. From the survey, we concluded that junior doctors do have some understanding of incident reviews process but they still do not feel comfortable with the idea of being under 'investigation'.

It is also important that formal support is made available during the process.

We organised a workshop in one of the 3 Trusts which was well attended and junior doctors asked if they could sit on review panels for experiential learning. This is to be presented to govenance teams across the mental health trusts in the region.

Further workshop across the 2 remaining Trusts could not be organised due to COVID-19 pandemic. 
An audit on prescribing practice and risk of serotonin syndrome among patients with chronic pain

Salakan Rai ${ }^{\star}$ and Aizad Yusof

Walsall Healthcare NHS Trust

${ }^{\star}$ Corresponding author.

doi: 10.1192/bjo.2021.425

Aims. To determine the incidence of prescribing practice with associated risk of serotonin toxicity among patients with chronic pain conditions.

Background. Serotonin syndrome is a potentially life-threatening condition caused by excessive serotonergic activity, usually from drug interactions. Concurrent use of antidepressants is strongly linked to serotonin syndrome, with recent data revealing record numbers of NHS prescribed antidepressants in 2019. Antidepressant medications are also used in chronic pain management for their anti-neuropathic pain properties. However, it is wellrecognised that a significant number of chronic pain patients suffer from anxiety and depression. This cohort of patients is therefore vulnerable to being exposed to multiple concurrent antidepressant agents, and thus at relatively higher risk of serotonin syndrome compared to other patient groups. Additionally, these patients are likely to be exposed to the concurrent use of antidepressants and certain analgesic agents particularly phenylpiperidine derivatives which increases serotonin toxicity risk.

Method. Medications of patients presenting to a secondary care pain clinic within the last year were looked into. Patients were selected at random by pain management secretaries. Concurrent use of multiple antidepressant agents including Selective Serotonin Reuptake Inhibitors (SSRIs), Serotonin Noradrenaline Reuptake Inhibitors (SNRIs), Tricyclic Antidepressants (TCAs) or Tetracyclic Antidepressant (TeCA) was noted. Additionally, concurrent use of any of these antidepressant agents and phenylpiperidine derivatives such as Fentanyl and Tramadol was noted. Result. Data on medications of 97 patients were collected. A total of 28 patients $(28.8 \%)$ were observed to have at-risk medication combinations. Out of these, five patients were on both SSRI and TCA. Two patients were on both TCA and TeCA. Four other patients were on either a combination of SSRI and SNRI, SNRI and TCA, SSRI and TeCA, or TCA and TCA. Three patients were on both Fentanyl patches and an antidepressant. Fourteen patients were on both an antidepressant and Tramadol. None of these patients were diagnosed with serotonin syndrome; however, it is unclear as to whether these patients experienced milder symptoms of the syndrome.

Conclusion. A considerable number of patients in this group were on medication combinations putting them at risk of serotonin syndrome. Despite no documented patient harm, there is an urgent need for an increased awareness among prescribers on drug interactions which may lead to this syndrome and a subsequent change in prescribing practice.

\section{A survey on psychiatry trainees' experiences of racism}

Aicha Rais ${ }^{\star}$, Richard Burton and Adeel Rauf

Derbyshire Healthcare NHS Foundation Trust

${ }^{*}$ Corresponding author.

doi: 10.1192/bjo.2021.426

Aims. To measure rates of racism experienced and witnessed by trainees training in Derbyshire.

Background. Derbyshire Healthcare Psychiatry trainee workforce comprises 39.1\% white, 52.2\% 'Black, Asian and Minority Ethnic'
(BAME) and $8.7 \%$ undisclosed ethnicity. Racism can affect trainees by increasing risk of poor mental health and psychological distress leading to worse health outcomes. Discrimination, marginalisation and segregation is related to poorer quality education and employment opportunities.

Method. Electronic surveys were sent out via e-mail to trainees in the North and South Derbyshire Hospital sites, accessible online from 11th to 31st December 2020.

Questions asked about personal experiences of racism, witnessing racism from patients and/or staff whilst training in Derbyshire. Trainees were asked if they know how to report incidents and if routinely reported. Trainee ethnicity was recorded.

Result. A total of 56 trainees received a survey request. Response uptake rate $25 \%$ (14 out of 56). Respondents comprised of $36 \%$ white and $64 \%$ BAME doctors. Over one third (36\%) of trainees reported experiencing racism from staff. $64 \%$ of trainees reported experience of racism from patients. There was no report of racism witnessed by staff towards patients. $29 \%$ of trainees reported witnessing both staff on staff racism and patient on staff racism. 93\% of trainees reported witnessing racism from patients to staff. $29 \%$ BAME trainees reported experiencing racism from both staff and patients. 7\% BAME trainees said they experienced racism from staff alone. $36 \%$ of trainees reported experiencing racism from patients only (4 BAME and 1 white trainee). $57 \%$ of trainees do not know how to report racism. 50\% of trainees said they would report racism if they knew how.

Conclusion. Racism remains a barrier affecting the lives of trainees requiring attention. BAME trainees are disproportionately affected by racism, and report witnessing more incidents, from staff and patients in the workplace. There remains an apprehension by doctors to report racism. A departmental presentation has been delivered on racism, unconscious bias, incident reporting process and sources of support. A workshop with the 'Equality Diversity Inclusion' team has been delivered to all trainees with the presence of the Freedom to Speak Up Guardian. Our British Medical Association Local Negotiating Committee Representative has also been informed.

\section{A national CASC course}

Yathooshan Ramesh* and Lisanne Stock

Barnet, Enfield and Haringey NHS Mental Health Trust

${ }^{*}$ Corresponding author.

doi: 10.1192/bjo.2021.427

Aims. There was understandable anxiety from trainees about the transition to the online format of the CASC due to the pandemic. There is also significant variability between trusts in the availability of lectures tailored specifically to the CASC exam. Having recent experience of the CASC exam, including the online format, we developed a free online lecture series. We aimed to address common questions relating to the exam, and selected topics that trainees may find daunting or had less experience with through clinical care. The topics covered were: An Introduction to the CASC, Mental State Examinations, Psychological Therapies, Pharmacology and a Q\&A Session.

Method. The course was designed to tackle areas that trainees often find difficult based on our own experiences as well as surveying course attendees. Prior to the course, we liaised with consultant site tutors \& junior doctor representatives to integrate the course into the local academic programme, and to facilitate promotion of the session to trainees across sites. We subsequently offered registration to trainees nationally. The course was planned and delivered by the organisers through interactive lecture-based 